\title{
Hot prominence detected in the core of a coronal mass ejection
}

\section{Analysis of the $\mathrm{C}$ III line detected by SOHO/UVCS}

\author{
S. Jejčič ${ }^{1,2}$, R. Susino ${ }^{3}$, P. Heinzel ${ }^{1}$, E. Dzifčáková ${ }^{1}$, A. Bemporad ${ }^{3}$, and U. Anzer ${ }^{4}$ \\ 1 Astronomical Institute, The Czech Academy of Sciences, 25165 Ondřejov, Czech Republic \\ 2 Faculty of Mathematics and Physics, University of Ljubljana, 1000 Ljubljana, Slovenia \\ e-mail: sonja.jejcic@guest.arnes.si \\ 3 INAF-Turin Astrophysical Observatory, 10025 Pino Torinese (TO), Italy \\ ${ }^{4}$ Max-Planck-Institut für Astrophysik, Karl-Schwarzschild-Str. 1, 85740 Garching bei München, Germany
}

Received 13 June 2017 / Accepted 11 August 2017

\begin{abstract}
Context. We study the physics of erupting prominences in the core of coronal mass ejections (CMEs) and present a continuation of a previous analysis.

Aims. We determine the kinetic temperature and microturbulent velocity of an erupting prominence embedded in the core of a CME that occurred on August 2, 2000 using the Ultraviolet Coronagraph and Spectrometer observations (UVCS) on board the Solar and Heliospheric Observatory (SOHO) simultaneously in the hydrogen L $\alpha$ and C III lines. We develop the non-LTE (departures from the local thermodynamic equilibrium - LTE) spectral diagnostics based on $\mathrm{L} \alpha$ and $\mathrm{L} \beta$ measured integrated intensities to derive other physical quantities of the hot erupting prominence. Based on this, we synthesize the $\mathrm{C}$ III line intensity to compare it with observations. Methods. Our method is based on non-LTE modeling of eruptive prominences. We used a general non-LTE radiative-transfer code only for optically thin prominence points because optically thick points do not allow the direct determination of the kinetic temperature and microturbulence from the line profiles. The input parameters of the code were the kinetic temperature and microturbulent velocity derived from the $\mathrm{L} \alpha$ and $\mathrm{C}$ III line widths, as well as the integrated intensity of the $\mathrm{L} \alpha$ and $\mathrm{L} \beta$ lines. The code runs in three loops to compute the radial flow velocity, electron density, and effective thickness as the best fit to the $\mathrm{L} \alpha$ and $\mathrm{L} \beta$ integrated intensities within the accuracy defined by the absolute radiometric calibration of UVCS data.

Results. We analyzed 39 observational points along the whole erupting prominence because for these points we found a solution for the kinetic temperature and microturbulent velocity. For these points we ran the non-LTE code to determine best-fit models. All models with $\tau_{0}(\mathrm{~L} \alpha) \leq 0.3$ and $\tau_{0}(\mathrm{C}$ III $) \leq 0.3$ were analyzed further, for which we computed the integrated intensity of the C III line using a two-level atom. The best agreement between computed and observed integrated intensity led to 30 optically thin points along the prominence. The results are presented as histograms of the kinetic temperature, microturbulent velocity, effective thickness, radial flow velocity, electron density, and gas pressure. We also show the relation between the microturbulence and kinetic temperature together with a scatter plot of computed versus observed C III integrated intensities and the ratio of the computed to observed C III integrated intensities versus kinetic temperature.

Conclusions. The erupting prominence embedded in the CME is relatively hot with a low electron density, a wide range of effective thicknesses, a rather narrow range of radial flow velocities, and a microturbulence of about $25 \mathrm{~km} \mathrm{~s}^{-1}$. This analysis shows a disagreement between observed and synthetic intensities of the C III line, the reason for which most probably is that photoionization is neglected in calculations of the ionization equilibrium. Alternatively, the disagreement might be due to non-equilibrium processes.
\end{abstract}

Key words. line: formation - radiative transfer - Sun: coronal mass ejections (CMEs) - techniques: spectroscopic Sun: UV radiation

\section{Introduction}

Solar prominences are well known as relatively cool and dense plasma structures with core temperatures of between 6000 and $10000 \mathrm{~K}$, with an electron density of $10^{10}-10^{11} \mathrm{~cm}^{-3}$, and with a gas pressure of $0.1-1 \mathrm{dyn}^{-2}$ (Labrosse et al. 2010; Vial \& Engvold 2015). They are located in the solar corona at altitudes of thousands of kilometers and are kept there against the solar gravity by magnetic fields. The magnetic field plays an important role in the stability of the prominence. Prominences are most probably formed around flux ropes, in the magnetized structure that often erupts as a result of the magnetic instability, and which then moves outward from the Sun with velocities reaching several hundred $\mathrm{km} \mathrm{s}^{-1}$ (Webb 2015). Erupting prominences are well observed with space coronagraphs and with other imaging instruments. The analysis of one eruptive prominence structure observed by the Ultraviolet Coronagraph and Spectrometer (UVCS) on board the Solar and Heliospheric Observatory (SOHO) in the hydrogen $\mathrm{L} \alpha$ line and by the Large Angle and Spectrometric Coronagraph (LASCOC2) in visible light (VL) on August 2, 2000 shows that the eruptive prominence embedded in the core of the coronal mass ejection (CME) reaches temperatures of about $10^{5} \mathrm{~K}$. Therefore it can be identified as a hot prominence structure with a low electron density of about $10^{8} \mathrm{~cm}^{-3}$ and a low gas pressure that is due to the expected expansion with velocities of several hundred $\mathrm{km} \mathrm{s}^{-1}$ (Heinzel et al. 2016, hereafter Paper I). Similar results were obtained by Pagano et al. (2014) using numerical magnetohydrodynamic (MHD) simulations of a CME with a flux rope inside. The authors proposed that the 
heating mechanisms might be reconnection and/or local plasma compression.

We here first combine the $\mathrm{L} \alpha$ and $\mathrm{C}$ III line observations that were simultaneously obtained by SOHO/UVCS in order to derive the kinetic temperature and the microturbulence (see, e.g., Tandberg-Hanssen 1995). We note that in Paper I these two parameters could not be derived and we therefore assumed an analytical relation between them according to Heinzel \& Anzer (2001). In this paper we extend our non-LTE modeling to pairs of $\mathrm{L} \alpha$ and $\mathrm{L} \beta$ lines that were simultaneously observed by UVCS and present a new way of fitting the observed and synthetic spectra. For prominence points that are optically thin in the $\mathrm{L} \alpha$ line, the total energy of the C III line is computed and compared with observations.

In Sect. 2 we present the C III line observations made with $\mathrm{SOHO} / \mathrm{UVCS}$ and the data reduction and calibration of the $\mathrm{C}$ III spectra. Section 3 considers Doppler velocities of simultaneously observed $\mathrm{L} \alpha, \mathrm{L} \beta$, and $\mathrm{C}$ III lines in order to demonstrate that all lines are formed in about the same plasma volume. Section 4 presents the kinetic temperature and microturbulence determination from the line widths of the L $\alpha$ and C III lines. Section 5 details the non-LTE modeling of the $\mathrm{L} \alpha$ and $\mathrm{L} \beta$ lines that provide the prominence parameters. Section 6 describes the CHIANTI modeling and coronal approximation for the $\mathrm{C}$ III line in the optically thin regime and shows that the radiative excitation is not negligible for low electron densities. In Sect. 7 we compare the models and spectroscopic observations of the $\mathrm{C}$ III line. Conclusions and future prospects are discussed in Sect. 8 .

\section{Observations and data reduction of the $\mathrm{C}$ III line}

We refer to Sect. 2 of Paper I for a detailed description of the analysis of LASCO-C2 VL and UVCS L $\alpha$ observations of the erupting prominence detected in the core of a CME that occurred on August 2, 2000 starting at 16:00 UT.

Here we recall that UVCS acquired spectra of several UV coronal lines as the erupting prominence crossed its field of view (FOV), a 40 arcmin long slit located perpendicularly to the solar radius at a latitude of $40^{\circ} \mathrm{NE}$ and at a heliocentric distance of $2.3 R_{\odot}$. Table 1 lists the lines that were detected, together with the formation temperature $\left(T_{\text {form }}\right)$ of the emitting ion according to the ionization balance of Bryans et al. (2006) and other parameters relevant to UVCS observations. All the spectral line profiles were acquired with a spatial resolution of 21 arcsec, a spectral resolution of about $0.2 \AA$, and an integration time of $120 \mathrm{~s}$ during the prominence eruption. We extend our investigation and the modeling presented in Paper I to the C III line observed by UVCS, and we also use the $\mathrm{L} \beta$ line to constrain the models (see Sect. 5).

$\mathrm{L} \alpha$ and $\mathrm{L} \beta$ data were calibrated using the standard calibration procedure provided by the UVCS Data Analysis Software (DAS; version 51); however, for the calibration of the C III line, we had to follow a different approach. The standard calibration of the DAS includes the correction of a distortion of the spectra that is caused by the fact that the crossed delay line (CDL) readout of the detector is not accurate near the corners of the readout area. This correction works fine for lines that are focused in the central part of the detector (such as the O VI or the H I Lyman lines), while we noted that it introduces artifacts in the spectral profiles of the lines that are located close to the detector edges (such as the C III line), in turn causing an anomalous line broadening in some regions along the UVCS FOV that is not physical.
Table 1. Lines identified in UVCS data.

\begin{tabular}{lllll}
\hline \hline Line & $\begin{array}{l}\log T_{\text {form }^{a}}{ }^{a} \\
(\mathrm{~K})\end{array}$ & $\begin{array}{l}\lambda_{0}{ }^{b} \\
(\AA)\end{array}$ & $\begin{array}{l}\lambda_{\text {ref }}^{c} \\
(\AA)\end{array}$ & $\begin{array}{l}\text { Spectral resolution } \\
(\AA)\end{array}$ \\
\hline C III & 4.9 & 977.02 & 976.51 & 0.198 \\
H I L $\beta$ & 4.5 & 1025.72 & 1025.76 & 0.198 \\
O VI & 5.5 & 1031.91 & & 0.198 \\
C II & 4.6 & 1036.34 & & 0.198 \\
C II & 4.6 & 1037.02 & & 0.198 \\
O VI & 5.5 & 1037.61 & & 0.198 \\
H I L $\alpha$ & 4.5 & 1215.67 & 1215.81 & 0.183 \\
\hline
\end{tabular}

Notes. ${ }^{(a)}$ Formation temperature of the line. ${ }^{(b)}$ Rest wavelength of the transition. ${ }^{(c)}$ Line reference wavelength (see text).

Since this broadening corrupts the results concerning the line widths, in the case of C III data we preferred to skip this step of the standard calibration and instead directly applied the radiometric calibration to the raw data using the radiometric factor provided by the DAS.

In order to remove the contribution to the line emission from the surrounding quiet corona, we subtracted from each line profile a pre-event profile of the same line averaged over the 50 exposures acquired before the appearance of the prominence in the UVCS FOV. The pre-event profile was also used to measure the line reference central wavelength $\lambda_{\text {ref }}$ (not necessarily equal to the laboratory rest central wavelength $\lambda_{0}$ ), needed to compute the Doppler shift and the line-of-sight (LOS) plasma velocity. These parameters are listed in Table 1 for the lines considered in this work.

As we pointed out in Paper $\mathrm{I}$, the $\mathrm{L} \alpha$ and $\mathrm{L} \beta$ lines are systematically formed by two components with different line widths. The two components were separated by fitting a doubleGaussian function to the line profiles; the consistency and significance of the fit was verified with a minimum $\chi$-squared analysis, as discussed in Sect. 2.2 of Paper I. In our interpretation, the narrow component (corresponding to temperatures of about $10^{5} \mathrm{~K}$ ) originates from the cool prominence plasma, while the broad component $(\sim 3.5 \mathrm{MK})$ is most probably produced by the plasma in the prominence-corona transition region (PCTR; see Parenti 2015) or, alternatively, in the hot shroud surrounding it (see Habbal et al. 2010, and the discussion in Paper I). Conversely, C III spectra do not show any signature of multiple components because the emission in this line is exclusively due to the cool prominence plasma. They were therefore fit with a singleGaussian function. In order to show that the broad (hot) component is not visible in the C III line, we estimated the ratio of the integrated intensity emitted in the $\mathrm{C}$ III line in the corona with a temperature of $10^{6} \mathrm{~K}$ and in the hot prominence with a temperature of $10^{5} \mathrm{~K}$ by assuming the coronal approximation (see Sect. 6.1). The ratio is on the order of $10^{-7}$, which indicates that the hot prominence plasma practically does not emit radiation in the C III line. We note that all parameters were computed from the CHIANTI database. For the statistical analysis presented in this work, we considered a sample of 68 points, selected all along the prominence, extracted from the sample used in Paper I for modeling the $\mathrm{L} \alpha$ line. We note that point 69 is not taken into consideration here because it is too noisy in the C III line.

The integrated intensities were derived for the three lines, and they are plotted in Fig. 1 as functions of time and latitude along the UVCS slit. 

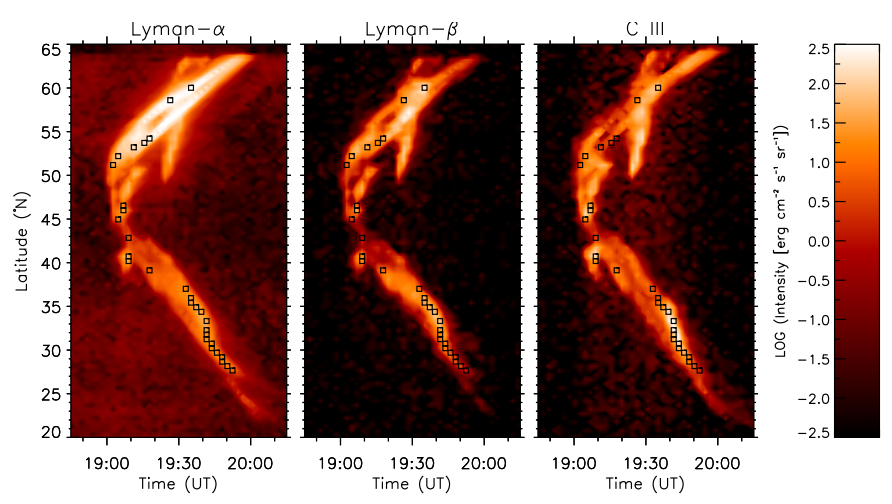

Fig. 1. Intensity distribution of the narrow components of the $\mathrm{L} \alpha$ and $\mathrm{L} \beta$ lines, and of the $\mathrm{C}$ III line, plotted as a function of time and latitude along the UVCS slit. The locations of the 30 optically thin points we chose for the analysis are marked with squares.

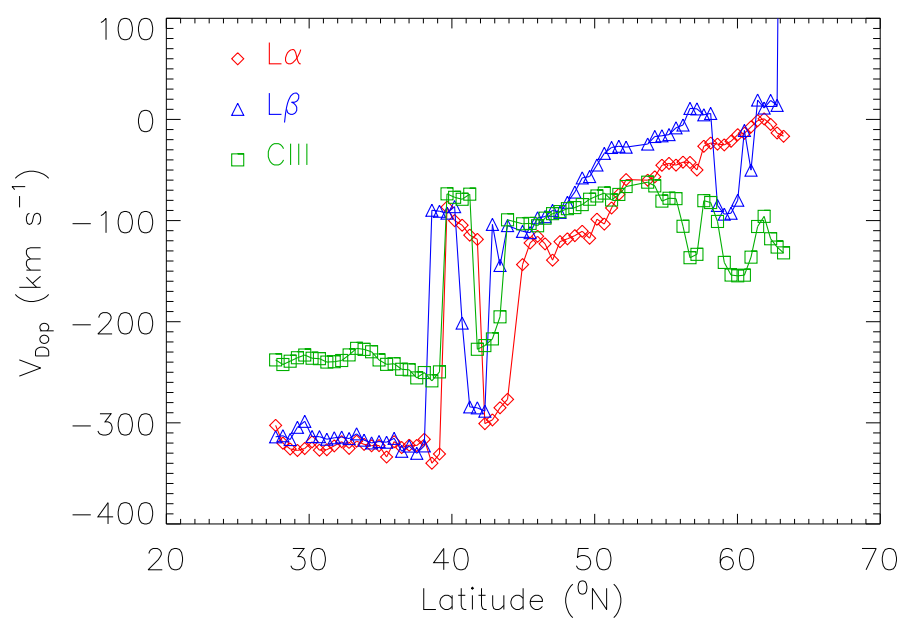

Fig. 2. Doppler velocities of the $\mathrm{L} \alpha, \mathrm{L} \beta$, and $\mathrm{C}$ III lines as a function of latitude along the UVCS slit. Note that the last three points of the $\mathrm{L} \beta$ line reach Doppler velocities of several hundred $\mathrm{km} \mathrm{s}^{-1}$.

\section{Doppler shifts of the C III line}

In our study of the $\mathrm{L} \alpha$ line, the kinetic temperature and the microturbulence were related according to Heinzel \& Anzer (2001) (see Paper I, Appendix A). Therefore, the obvious next step was to use the L $\alpha$ and C III lines to distinguish between kinetic temperature and microturbulence. This is only possible when two species with different masses are formed in the same emitting plasma volume (Tandberg-Hanssen 1995) and are both optically thin. In this section we check whether this condition is met in the spectroscopic UVCS observations of the $\mathrm{L} \alpha, \mathrm{L} \beta$, and C III lines.

We present a detailed Doppler velocity analysis of all the 68 points we measured along the prominence in the $\mathrm{L} \alpha, \mathrm{L} \beta$, and $\mathrm{C}$ III lines in Fig. 2. This shows that for the majority of the points, the Doppler velocities of the C III line are consistent with the $\mathrm{L} \alpha$ and $\mathrm{L} \beta$ velocities within the uncertainty of the UVCS wavelength determination, which is equal to the spectral UVCS resolution of about $0.2 \AA$. This gives an uncertainty in the Doppler velocity of about $60 \mathrm{~km} \mathrm{~s}^{-1}$. The Doppler velocities of the three lines are almost consistent within the estimated uncertainties. $\mathrm{L} \alpha$ and $\mathrm{L} \beta$ lines must have the same Doppler velocities because they are emitted from the hydrogen atoms. From this we conclude that the C III ions also move with the same Doppler velocity as hydrogen. Therefore, the $\mathrm{L} \alpha, \mathrm{L} \beta$ and $\mathrm{C}$ III lines are formed in the same or a similar emitting plasma volume with a unique kinetic

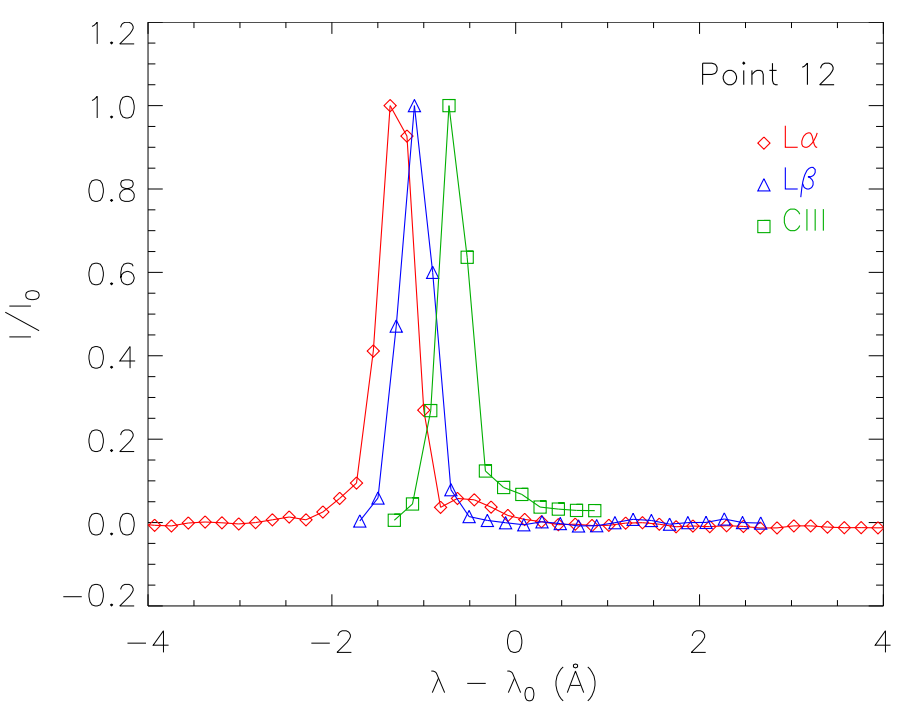

Fig. 3. Profiles of the $\mathrm{L} \alpha, \mathrm{L} \beta$, and $\mathrm{C}$ III lines of the 12 th measured point along the erupting prominence.

temperature and microturbulent velocity. In addition, firm proof that all lines are formed in the same plasma volume is provided by the fact that the profiles of all three lines, reported in Fig. 3 in one representative case, exhibit a single peak. We note that their Doppler shifts should be proportional to $\lambda_{0}$. If the profiles had more than one peak, it would indicate that the prominence blobs move with different velocities in different directions, and this is not observed.

\section{Kinetic temperature and microturbulent velocity}

The line profiles of optically thin prominences are mainly Doppler broadened as a result of the thermal motions of atoms and because of the turbulent plasma motions. The Doppler line width can then be expressed as

$\Delta \lambda_{\mathrm{D}}=\frac{\lambda_{0}}{c} \sqrt{\frac{2 k_{\mathrm{B}} T}{m}+\xi^{2}}=\frac{\lambda_{0}}{c} \sqrt{\frac{2 k_{\mathrm{B}} T_{\mathrm{eff}}}{m}}$.

Here $c$ is the speed of light, $k_{\mathrm{B}}$ is the Boltzmann constant, $T$ is the kinetic temperature, $m$ is the mass of the atom, $\xi$ is the microturbulent velocity, and $T_{\text {eff }}$ is the effective temperature. We introduced the microturbulence because in erupting prominences that are embedded in CME cores, significant nonthermal motions are expected to occur. Equation (1) shows that the carbon line is more sensitive to the microturbulent velocity and less sensitive to the kinetic temperature, as is clearly shown in Fig. 4.

The kinetic temperature and the microturbulent velocity of the prominence structure were computed from the Gaussian line widths of the hydrogen L $\alpha$ and C III lines using Eq. (1). We assumed that the two lines of ions with different masses are formed in the same emitting plasma volume (Sect. 3) and are optically thin. We note that optically thick lines exhibit the so-called opacity broadening and cannot be used in the method described here (the elimination of such pixels is described below). The kinetic temperature can be expressed as

$T=\frac{T_{\mathrm{eff}}^{(2)} m_{1}-T_{\mathrm{eff}}^{(1)} m_{2}}{m_{1}-m_{2}}$,

where $m_{1}$ and $m_{2}$ are the masses of the hydrogen and carbon atoms, respectively, and $T_{\mathrm{eff}}^{(1)}$ and $T_{\mathrm{eff}}^{(2)}$ are the effective temperatures of the L $\alpha$ and C III lines that are calculated from the Gaussian line widths according to Eq. (1) after deconvolution of the 


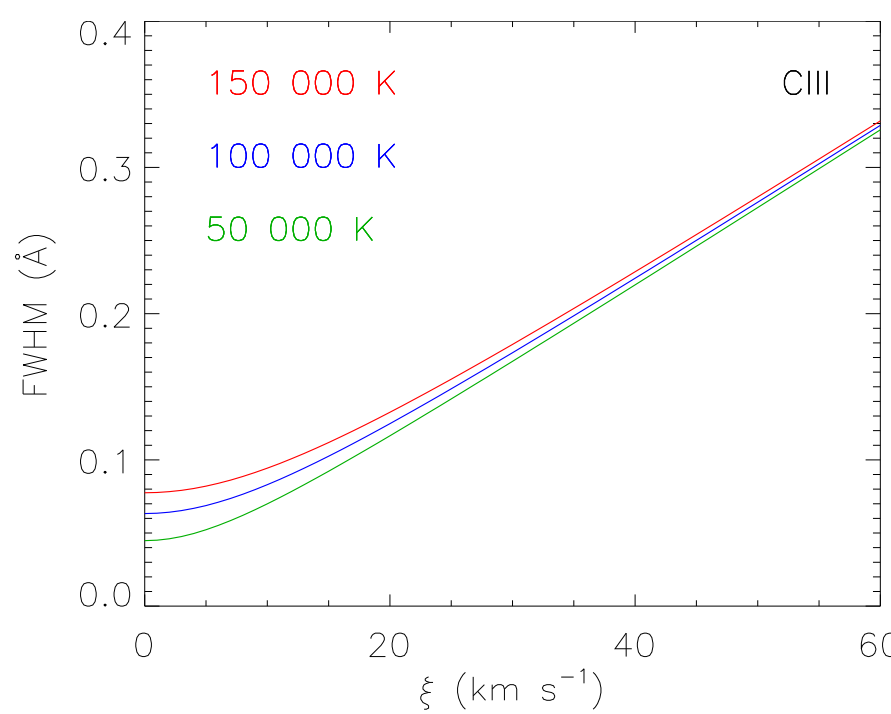

(a)

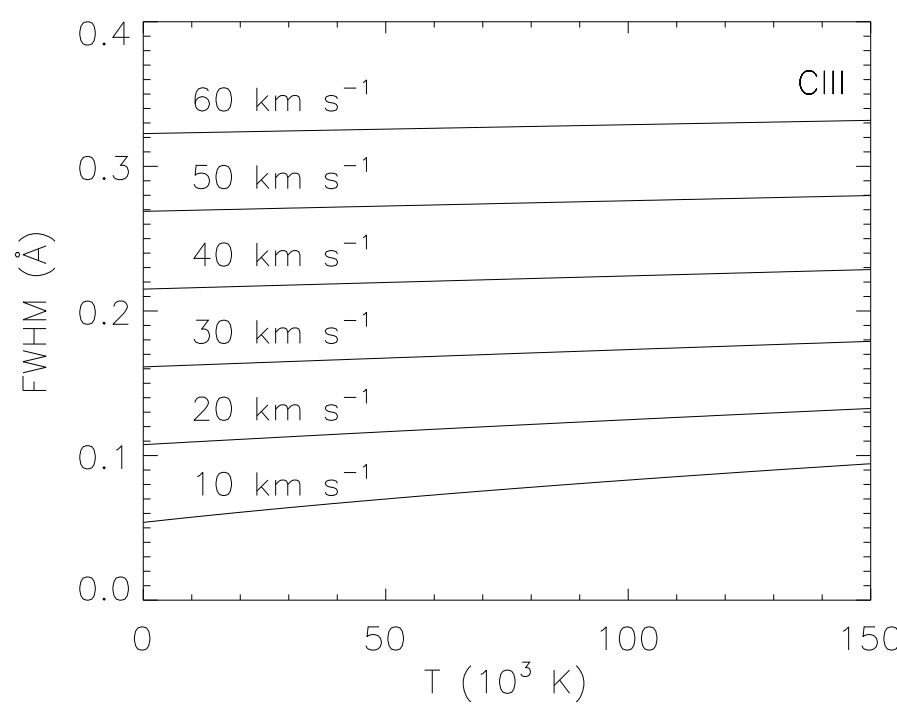

(b)

Fig. 4. Panel $a$ : FWHM of the C III line plotted as a function of the microturbulent velocity for three typical kinetic temperatures. Note that the $F W H M=2 \sqrt{\ln 2} \Delta \lambda_{\mathrm{D}}$. Panel $b$ : FWHM of the C III line as a function of the kinetic temperature for six microturbulent velocities.

UVCS instrumental profile. The microturbulent velocity is then given by

$\xi=\sqrt{\frac{2 k_{\mathrm{B}}}{m}\left(T_{\mathrm{eff}}-T\right)}$

where $m$ and $T_{\text {eff }}$ belong to one of the observed lines. Therefore, having observed two optically thin lines from two species with substantially different masses, we are in principle able to derive both the kinetic temperature and the microturbulent velocity. We note that in Paper I we assumed that the microturbulent velocity is equal to one-half of the sound speed.

Kinetic temperature and microturbulent velocity were computed using Eqs. (2) and (3) for all 68 measured points along the prominence. We restricted ourselves to positive microturbulent velocity and to kinetic temperatures higher than $20000 \mathrm{~K}$. At these temperatures, hydrogen is significantly ionized and the Lyman lines are moderately optically thick or become thin at

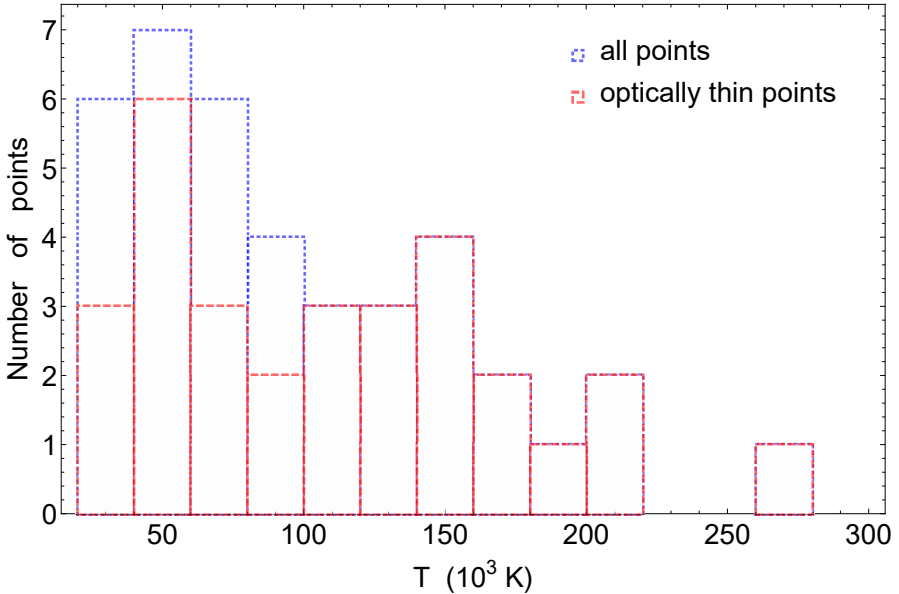

Fig. 5. Kinetic temperature distribution of all 39 studied points along the prominence (dotted blue line). The kinetic temperature distribution of all 30 selected points along the prominence in the optically thin regime is shown by the dashed red line.

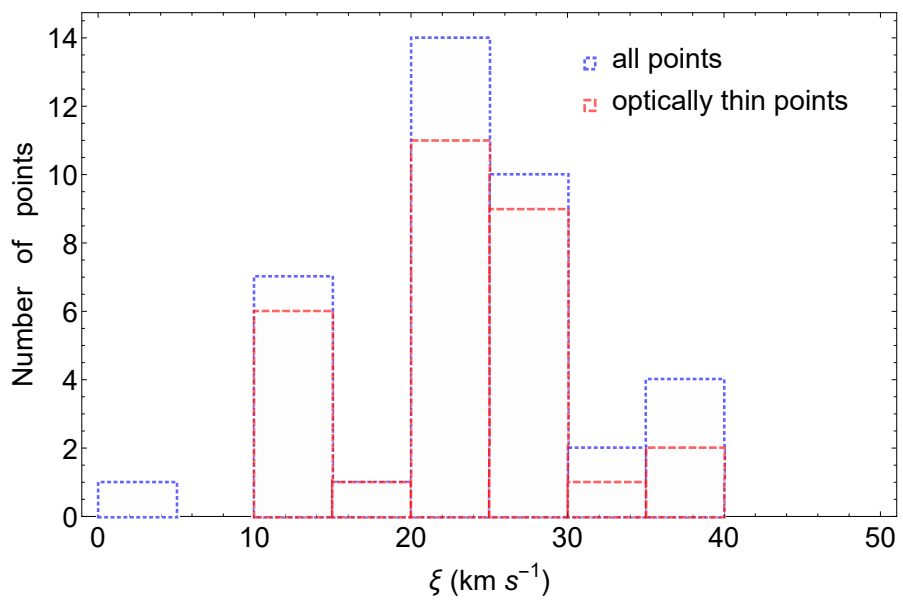

Fig. 6. Microturbulence distribution of all 39 studied points along the prominence (dotted blue line). The microturbulent velocity distribution of all 30 selected points along the prominence in the optically thin regime is shown by the dashed red line.

higher temperatures. For lower temperatures their optical thickness rapidly increases, and this situation would require a special numerical treatment. In total, these two conditions narrow the sample down to 39 points. In Fig. 5 we show the distribution of the kinetic temperature for all these points with a dotted blue line. The majority of them have a kinetic temperature of between 50000 and $200000 \mathrm{~K}$. The mean weighted kinetic temperature is $99000 \mathrm{~K}$. The distribution of the microturbulent velocity for the same points, marked by dotted blue line, is shown in Fig. 6. Most of the points have a microturbulent velocity of between 20 and $30 \mathrm{~km} \mathrm{~s}^{-1}$, while the mean weighted microturbulent velocity is $23 \mathrm{~km} \mathrm{~s}^{-1}$. While Landi et al. (2010) suggested that the nonthermal line widths provide a limit on wave heating in eruptive plasma, additional nonthermal broadening can be due to the expansion of the prominence flux tube, as discussed elsewhere in this paper.

\section{Non-LTE modeling of the hydrogen Lyman lines}

In Paper I we used the multilevel hydrogen code MALI to find best-fit models of the hot erupting prominence. The models were constrained by the integrated intensity of the hydrogen $\mathrm{L} \alpha$ line, 
and the MALI code was run through the loop in which the gas pressure increased up to the value that caused the synthetic line intensity to be consistent with the UVCS observation in a given pixel. This also gave us the electron density $n_{\mathrm{e}}$. Kinetic temperature and microturbulent velocity have been obtained from the Gaussian line width by assuming that the mean microturbulent velocity is a fraction (one-half) of the sound speed - see Appendix A of Paper I. Other input parameters were estimated from UVCS observations, such as the height above the solar surface, the effective thickness $D_{\text {eff }}$, and the radial flow velocity $v_{\text {rad. }}$. For the effective thickness we used a characteristic value of $1000 \mathrm{~km}$, and the radial velocity was estimated from plane-ofsky (POS) measurements of the prominence time evolution and from Doppler shifts of the $\mathrm{L} \alpha$ line. We modeled all the 69 pixels in this way. In two pixels, however, we were able to apply a more realistic modeling because we also had LASCO-C2 visible light intensities, which directly provided the column density of electrons. For these two pixels we also expanded our fitting to the $\mathrm{L} \beta$ line (see Table 2 in Paper I).

In the present work we used a different approach on the full calibrated data set of three spectral lines, that is, the hydrogen $\mathrm{L} \alpha, \mathrm{L} \beta$, and $\mathrm{C}$ III lines observed by UVCS. As described in Sect. 4, the L $\alpha$ and C III lines were used to obtain the kinetic temperature and microturbulent velocity using the standard method that is valid for two optically thin lines formed in the same plasma volume. The results are similar to those shown in Fig. 11 in Paper I. For 39 pixels we found the solution for the kinetic temperature and the microturbulence, and those pixels were used for a detailed non-LTE modeling to find the best-fit models capable of reproducing the observational results and also to verify which models truly are optically thin in $\mathrm{L} \alpha(\mathrm{L} \beta$ is of course even thinner).

In principle, having three lines, we should be able to obtain three unknown plasma parameters. These are the electron density (or equivalently, the gas pressure), the prominence effective thickness, and the radial flow velocity. We note that here we did not use $v_{\text {rad }}$ as the parameter derived from other observations as in Paper I, but we have it as one of the unknown parameters to be found by the line fitting. This is the velocity at which the Doppler dimming effect (DDE), explained by Heinzel \& Rompolt (1987), in both Lyman lines is consistent with the DDE that acts for the real velocity vector. Since the $\mathrm{L} \alpha$ line is more sensitive to the DDE than $\mathrm{L} \beta$, these two lines provide a constraint on the radial flow velocity. The best fit to these hydrogen lines gives a set of models with various combinations of electron density and effective thickness. To determine the best-fit model, we finally used the C III line, which is most sensitive to electron density (for a given kinetic temperature). Details of the fitting are discussed below and in Sect. 7.

It is evident that the DDE is most sensitive to vertical (radial) motions, while it is much less sensitive to horizontal motions in the corona. The moving structures are illuminated by the solar disk radiation at a given height above the surface. In the case of the prominence analyzed here, the height is relatively large, around $950000 \mathrm{~km}$, so that the radial component of the outflow velocity is indeed dominant, and the Doppler velocities shown in Fig. 2 are not expected to play a significant role. In principle, it is possible to derive the true vector of the flow velocity from the POS motion and adding the Doppler velocity component from the spectra. However, in practice, various difficulties arise (see discussion in Paper I), and we therefore used the radial component of the flow velocity as a free parameter here, to which different lines have a different sensitivity via the DDE.
In order to determine best-fit models or to explore the space of all realistic models that are consistent with the observed $\mathrm{L} \alpha$ and $\mathrm{L} \beta$ line intensities, we proceeded in the following way. The MALI code for hydrogen described in Paper I was executed in three nested loops. The outermost loop covered the range of radial flow velocities from 80 to $460 \mathrm{~km} \mathrm{~s}^{-1}$ (with steps of $20 \mathrm{~km} \mathrm{~s}^{-1}$ ), the inner loop covered input electron densities ranging from $2 \times 10^{6}$ to $1.21 \times 10^{8} \mathrm{~cm}^{-3}$ (with steps of $10^{6} \mathrm{~cm}^{-3}$ ), and finally, the innermost loop covered the effective thickness range from 500 to $50000 \mathrm{~km}$ (with steps of $500 \mathrm{~km}$ ). These ranges were selected based on our previous experience (Paper I) and on the fact that the upper limit of $D_{\text {eff }}$ is $56000 \mathrm{~km}$ based on LASCO-C2 measurements. Altogether, we had up to 240000 models. These models cannot be precomputed as a unique grid because they critically depend on the kinetic temperature and also on the microturbulent velocity. In fact, when searching for the best fit in both Lyman lines, we discarded the solutions that led to a L $\alpha$ line optical thickness larger than 0.3 , and we therefore required fewer models to be computed. This significantly improved the speed of the whole modeling, although to cover all 39 pixels, the CPU time reaches tens of hours on a PC (note that the MALI code is based on the so-called ALI technique, which is numerically very efficient for solving the multilevel non-LTE problems). In this procedure we can also set the arbitrary range on the accuracy of the observed line intensities. The accuracy of the absolute radiometric calibration of UVCS is not better than about $30-40 \%$, and we therefore used a value of $40 \%$ to constrain the models. For each pixel we obtained a set of best-fit models that were then used to compute the $\mathrm{C}$ III line intensity as described in the next section.

\section{Modeling the $\mathrm{C}$ III line intensity}

\subsection{CHIANTI modeling}

For calculations of the C III level populations, we used CHIANTI 8.0 (Dere et al. 1997; Del Zanna et al. 2015) with atomic data from Berrington et al. (1985, 1989), Martin et al. (1995), and Tachiev \& Froese Fischer (1999). We used a 20-level C III atomic model. The ionization equilibrium was taken from Dere (2007). We note that photoionization and photoexcitation processes are not included in this calculation. We discuss the role of photoionization of C III to Sect. 7. However, as we show in Sect. 6.2, the radiative excitation (photoexcitation) in the C III line is not negligible, and we therefore took it into account in the framework of a simplified two-level atomic model because CHIANTI does not provide such an option in a straightforward way. Before we applied the two-level model, we checked how reliable it is in comparison with the multilevel CHIANTI solution, neglecting the photoexcitations. We are interested in the C III line transition (977.02 $\AA$ ) between the ground state and the excited state $2 \mathrm{~s} 2 \mathrm{p} 1 \mathrm{P}$, and in the two-level model atom, we denote these two levels by indexes 1 and 2, respectively.

For a range of electron temperatures from 10000 to $400000 \mathrm{~K}$ and an electron density from $10^{6}$ to $10^{10} \mathrm{~cm}^{-3}$ we computed, using the CHIANTI database, the collisional excitation coefficient $q_{12}(T)$, population ratio of the ground state ions relative to the total $\mathrm{C}$ III ions $n_{1} / n_{\mathrm{C} \text { III }}$, and the ratio of $\mathrm{C}$ III ions to the total number of carbon atoms $n_{\mathrm{C} \text { III }} / n_{\mathrm{C}}$. Best fits for these quantities are shown in Figs. 7-9. Figure 9 shows a characteristic formation temperature of the C III ion of between 70000 and $80000 \mathrm{~K}$, consistent with Table 1 . We note that we here assumed equality between electron and ion (kinetic) temperature. 


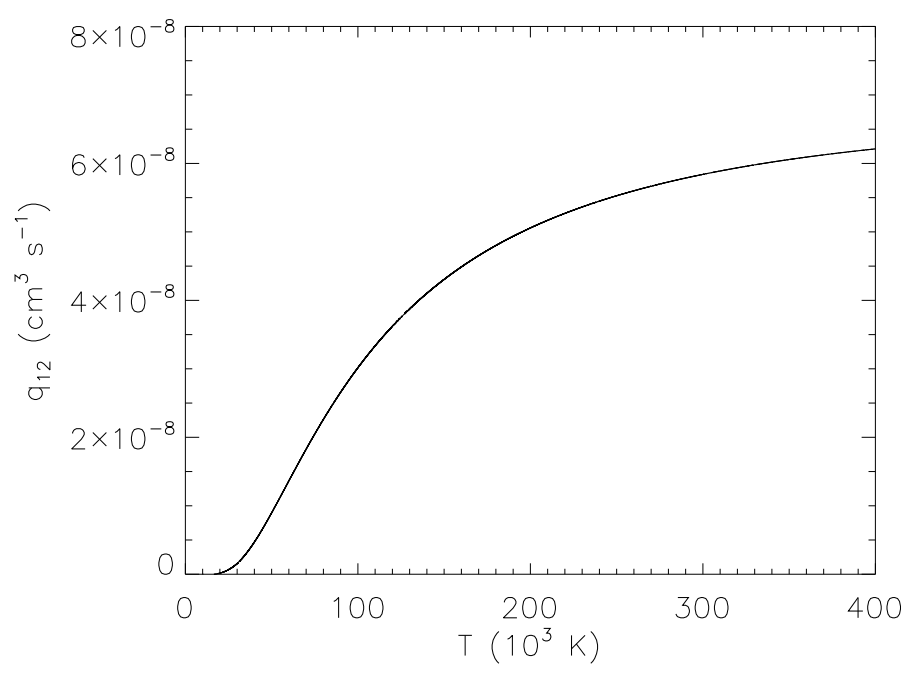

Fig. 7. Collisional excitation coefficient as a function of the electron temperature.

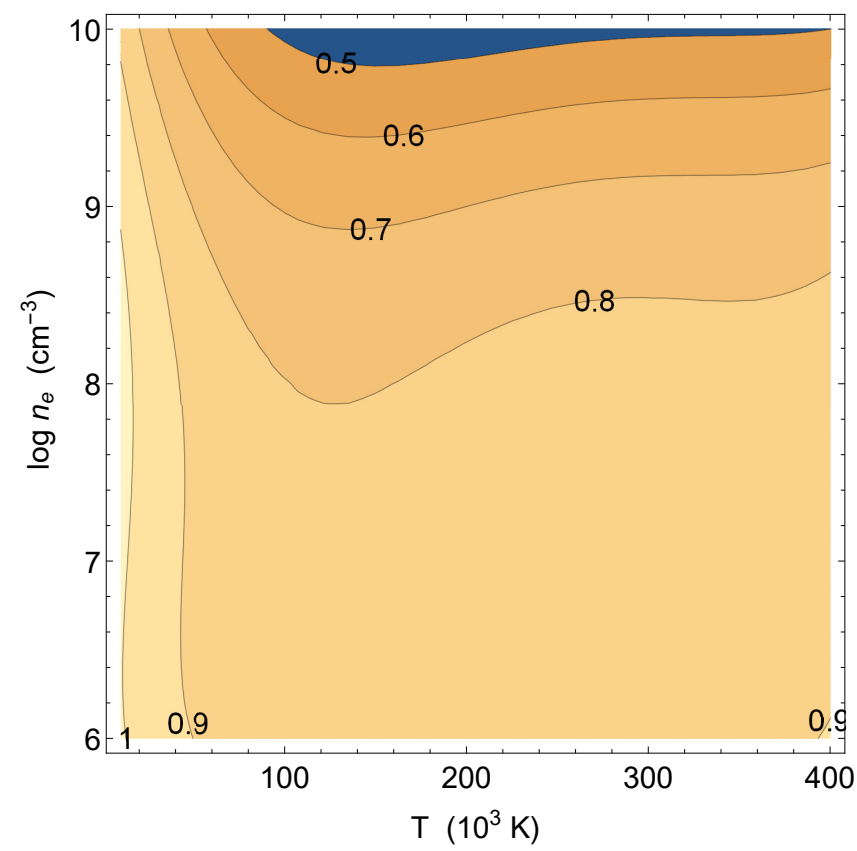

Fig. 8. Ratio $n_{1} / n_{\mathrm{C} \text { III }}$ as a function of the electron temperature and the electron density.

The statistical equilibrium equation for an approximate twolevel C III line formation assuming the coronal approximation can be written as

$n_{1} C_{12}(T)=n_{2} A_{21}$,

where the collisional excitation rate is $C_{12}(T)=n_{\mathrm{e}} q_{12}(T)$ (Hubeny \& Mihalas 2015). $q_{12}(T)$ is displayed in Fig. 7, $n_{1}$ is the number density of C III ions in the ground state, $n_{2}$ is the number density in the first excited state, and $A_{21}$ is the Einstein coefficient for spontaneous emission (CHIANTI gives $\left.A_{21}=1.769 \times 10^{9} \mathrm{~s}^{-1}\right)$. In the coronal approximation we neglected the radiative excitation rate, stimulated emission which is quite small in the $\mathrm{UV}$, and a collisional de-excitation rate that is lower than the spontaneous radiative rate on the right-handside of Eq. (4). $n_{1}$ is computed as

$n_{1}=\frac{n_{1}}{n_{\mathrm{C} \text { III }}} \frac{n_{\mathrm{C} \text { III }}}{n_{\mathrm{C}}} A_{\mathrm{C}} n_{\mathrm{H}}$,

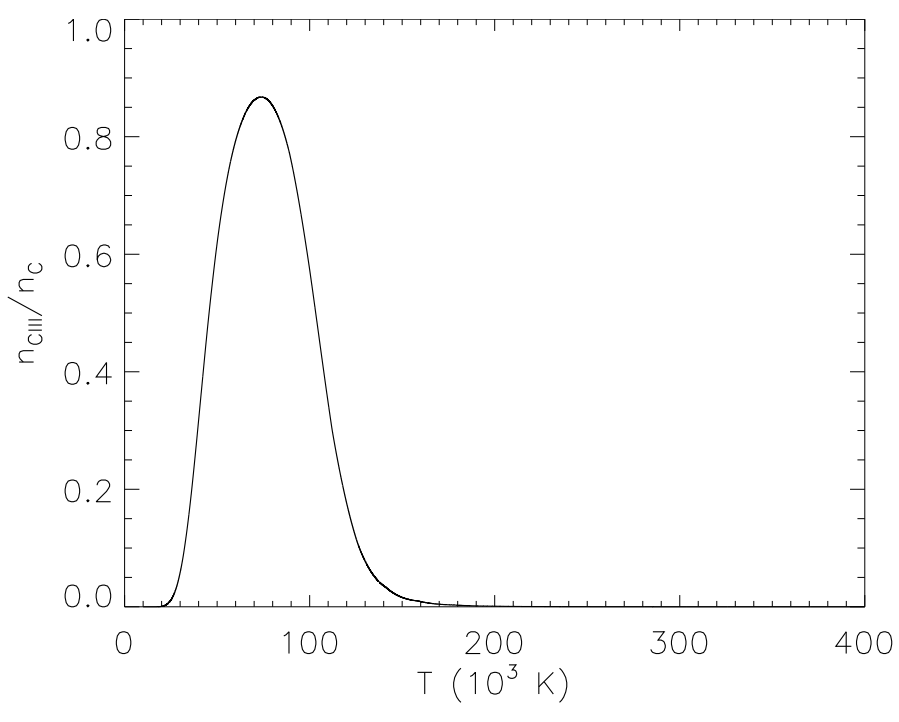

Fig. 9. Ratio $n_{\mathrm{C} \text { III }} / n_{\mathrm{C}}$ as a function of the electron temperature.

where $A_{\mathrm{C}}=n_{\mathrm{C}} / n_{\mathrm{H}}$ is the abundance of carbon relative to hydrogen $\left(A_{\mathrm{C}}=3.31131 \times 10^{-4}\right.$ from CHIANTI), and the first two ratios are derived from the plots shown in Figs. 8 and 9. The hydrogen is almost fully ionized at kinetic temperatures found in the eruptive prominence and therefore $n_{\mathrm{H}} \sim n_{\mathrm{e}}$ (here we neglect the helium contribution to $n_{\mathrm{e}}$ ). For optically thin structures, the integrated intensity emitted in the C III line can be generally expressed as

$E_{\mathrm{C} \text { III }}=n_{2} A_{21} \frac{h c}{\lambda_{0}} \frac{D_{\mathrm{eff}}}{4 \pi}$,

where $h$ is the Planck constant. Using Eq. (4), we finally obtained

$E_{\mathrm{C} \text { III }}=n_{1} C_{12}(T) \frac{h c}{\lambda_{0}} \frac{D_{\mathrm{eff}}}{4 \pi}$.

In Eqs. (4) and (7) we assumed that only the collisional excitation populates the level 2s $2 \mathrm{p} 1 \mathrm{P}$ (level 2). However, from the full 20-level CHIANTI solution we find that the level $2 \mathrm{~s} 2 \mathrm{p} 1 \mathrm{P}$ is far more sparsely populated than the metastable levels $2 \mathrm{~s} 2 \mathrm{p} 3 \mathrm{P}$, and the question arises whether the collisional excitations from these metastable levels can significantly populate the level $2 \mathrm{~s} 2 \mathrm{p} 1 \mathrm{P}$. We note that the decrease in fractional population of the ground state below unity, as shown in Fig. 8, is due to the significant population of metastable levels $2 \mathrm{~s} 2 \mathrm{p} 3 \mathrm{P}$. To test this, we used the full CHIANTI solution, which also contains the collisional excitations from metastable levels toward the $2 \mathrm{~s} 2 \mathrm{p} 1 \mathrm{P}$ level, and we compared the emissivity in the C III line computed by CHIANTI with that computed from the two-level approximation in Eq. (7). The relative difference as a function of the electron temperature and density is shown in Fig. 10. We clearly see that for the range of temperatures and densities found in the studied erupting prominence, the difference is below $10 \%$, and this justifies the application of the two-level model atom in the next sections. However, at higher temperatures and higher electron densities, the difference can reach more than $20 \%$. In other words, the additional population of the $2 \mathrm{~s} 2 \mathrm{p} 1 \mathrm{P}$ level by collisional excitations from metastable levels seems to be rather negligible in our case.

\subsection{Importance of the radiative excitation in C III line}

The mean integrated intensity of the incident solar disk radiation assuming a Doppler-shifted Gaussian absorption profile is 


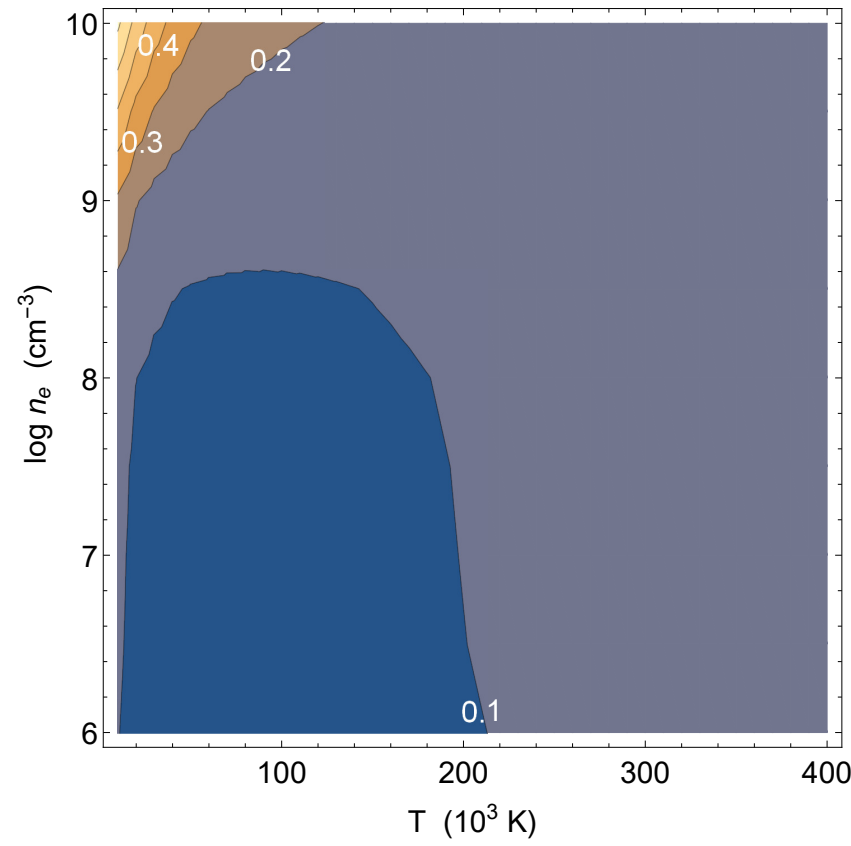

Fig. 10. Relative difference between the C III emissivity computed with CHIANTI and with the two-level atom as a function of the electron temperature and the electron density.

expressed as

$\bar{J}_{12}=\int_{-\infty}^{\infty} \frac{\lambda_{0}^{2}}{\sqrt{\pi} c \Delta \lambda_{\mathrm{D}}} e^{-\left(\frac{\Delta \lambda}{\Delta \lambda_{\mathrm{D}}}\right)^{2}} J(\lambda) \mathrm{d} \lambda$,

where $\Delta \lambda=\lambda-\lambda_{0}-\frac{\lambda_{0}}{c} v_{\text {rad }}$ is the wavelength redshift (we are interested in structures that move outward from the Sun) and depends on the radial flow velocity of the moving structure. $J(\lambda)$ is the mean intensity of the incident radiation from the solar surface. In UV the center-to-limb variation is negligible and $J(\lambda)$ is thus equal to the intensity emitted from the solar disk center $I(\lambda)$ multiplied by a geometrical dilution factor $W$, that is, $J(\lambda)=W I(\lambda) . W=0.047$ at a typical position of the UVCS slit at a height of $950000 \mathrm{~km}$ above the solar surface. The Solar Ultraviolet Measurements of Emitted Radiation (SUMER) spectrograph on board the SOHO spacecraft has measured the solar disk radiation between $670 \AA$ and $1609 \AA$ (Curdt et al. 2001). $I(\lambda)$ for the C III line (see the inset of the Fig. 11) is therefore derived from the SUMER spectral atlas.

To show the importance of the DDE for the C III line, we plot in Fig. $11 \bar{J}_{12}$ as a function of radial flow velocity. $\bar{J}_{12}$ is computed from Eq. (8) for a typical prominence plasma with a kinetic temperature of $10^{5} \mathrm{~K}$, a microturbulent velocity of $25 \mathrm{~km} \mathrm{~s}^{-1}$, and at a typical height of $950000 \mathrm{~km}$. The radial flow velocity ranges from zero to $300 \mathrm{~km} \mathrm{~s}^{-1}$. The plot clearly shows that for radial flow velocities on the order of $100 \mathrm{~km} \mathrm{~s}^{-1}$ and higher, the $\bar{J}_{12}$ term is negligible because of the DDE. We note that Gontikakis \& Vial (2016) also reported evidence of scattering effects in the $\mathrm{C}$ IV line.

The contribution of the radiative excitation term in the twolevel atom modifies Eqs. (4) and (7)

$$
\begin{aligned}
& n_{1}\left[R_{12}+C_{12}(T)\right]=n_{2} A_{21}, \\
& E_{\mathrm{C} \text { III }}=n_{1}\left[R_{12}+C_{12}(T)\right] \frac{h c}{\lambda_{0}} \frac{D_{\mathrm{ff}}}{4 \pi} .
\end{aligned}
$$

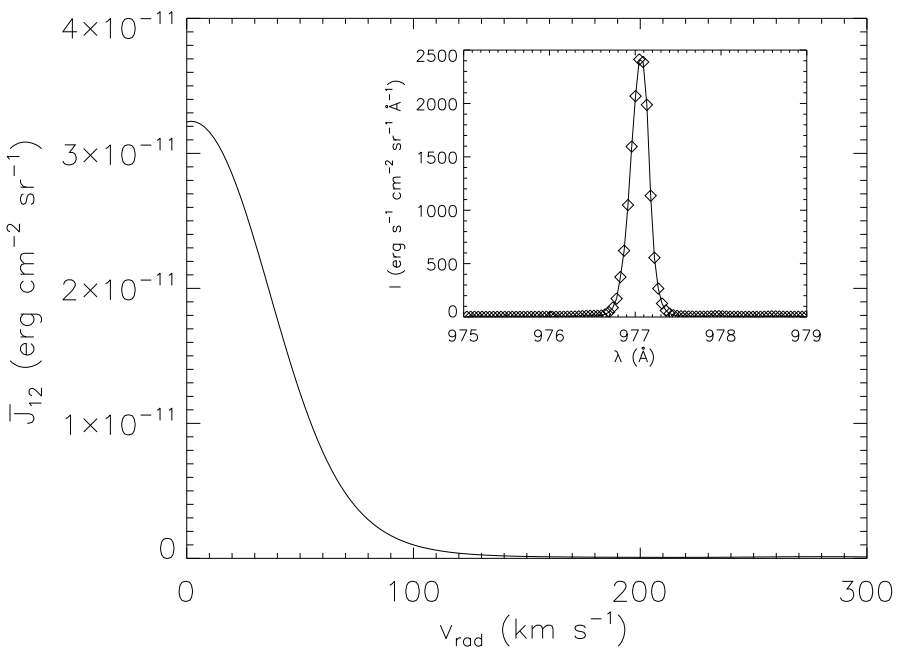

Fig. 11. Mean integrated intensity of the incident solar radiation as a function of the radial flow velocity for the $\mathrm{C}$ III line showing the importance of the DDE. In the inset the intensity emitted from the solar disk center is shown for the $\mathrm{C}$ III line as observed by SOHO/SUMER.

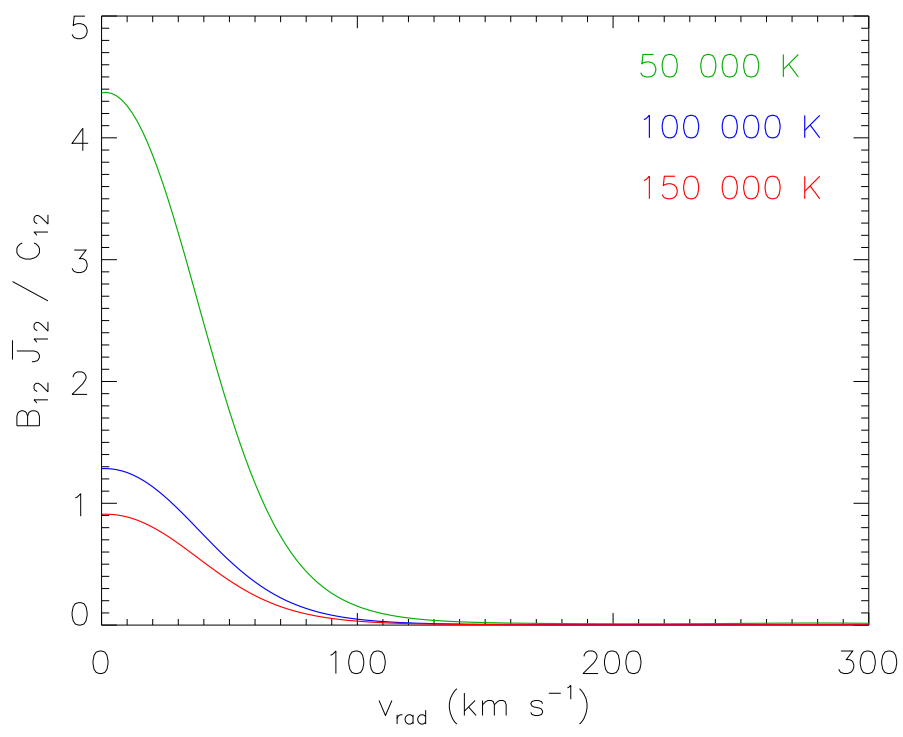

Fig. 12. Ratio $B_{12} \bar{J}_{12} / C_{12}$ as a function of the radial flow velocity at a microturbulent velocity of $25 \mathrm{~km} \mathrm{~s}^{-1}$, an electron density of $10^{7} \mathrm{~cm}^{-3}$, and at an altitude of $950000 \mathrm{~km}$ above the solar surface for three representative kinetic temperatures marked with different colors.

Here $R_{12}=B_{12} \bar{J}_{12}$ is the radiative excitation rate. CHIANTI gives the Einstein coefficient for absorption as $B_{12}=1.246 \times$ $10^{10} \mathrm{erg}^{-1} \mathrm{~s}^{-1} \mathrm{~cm}^{2} \mathrm{sr}$.

To demonstrate the role of the radiative excitation rate compared to the collisional excitation rate, we plot in Fig. 12 their ratio computed for plasma at three representative kinetic temperatures of 50000,100000 , and $150000 \mathrm{~K}$, a microturbulent velocity of $25 \mathrm{~km} \mathrm{~s}^{-1}$, an electron density of $10^{7} \mathrm{~cm}^{-3}$, and a height of the UVCS slit equal to $950000 \mathrm{~km}$, with different radial flow velocities of up to $300 \mathrm{~km} \mathrm{~s}^{-1}$. We see that the radiative excitation term is generally lower than collisional term, but for a low electron density and a radial flow velocity below $100 \mathrm{~km} \mathrm{~s}^{-1}$ it is not negligible. The estimate of Ko et al. (2005) leads to even higher radiative excitation rates in the $\mathrm{C}$ III line under similar prominence conditions. Because this radiative excitation term cannot be easily included in the CHIANTI modeling, we used Eq. (9) to compute the C III intensity. 


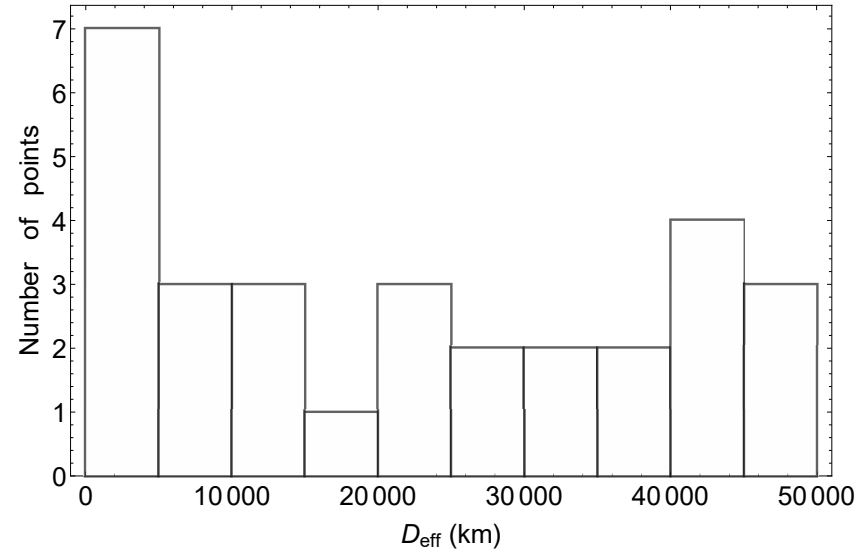

Fig. 13. Effective thickness distribution for all 30 selected points along the prominence in optically thin structures.

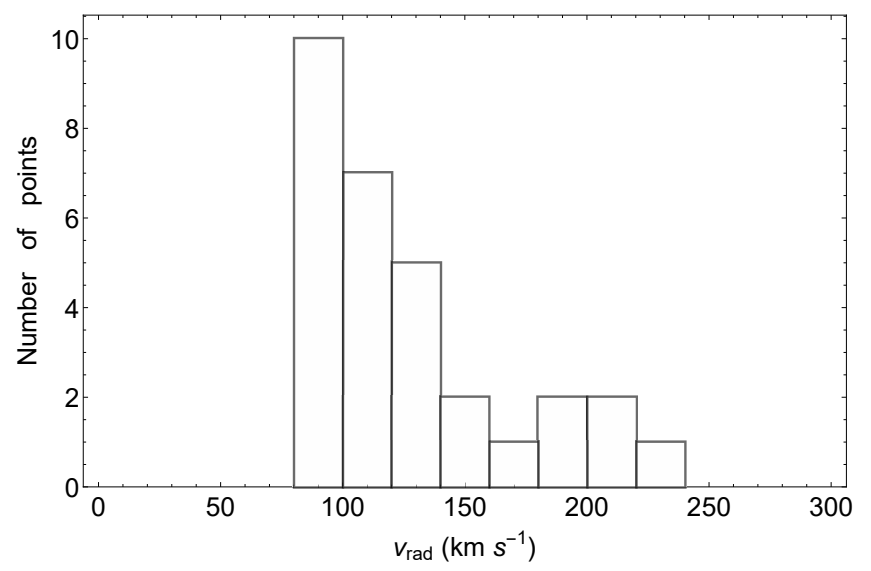

Fig. 14. Radial flow velocity distribution for all 30 selected points along the prominence in optically thin structures.

\section{Results of radiative-transfer modeling}

From all 39 pixels for which the kinetic temperature and microturbulent velocity were computed, we selected the points for which the $\mathrm{L} \alpha$ line was optically thin $\left(\tau_{0}(\mathrm{~L} \alpha) \leq 0.3\right)$. We still obtained hundreds or thousands of best-fit models because of the $40 \%$ uncertainty. They were then used to compute the synthetic $\mathrm{C}$ III line intensity to further constrain the model parameters. For this we used the two-level model described by Eq. (9). We first computed the C III line optical thickness at the line center in the following way:

$\tau_{0}(\mathrm{C}$ III $)=\frac{\sqrt{\pi} e^{2}}{m_{\mathrm{e}} c^{2}} f_{12} n_{1} \frac{\lambda_{0}^{2}}{\Delta \lambda_{\mathrm{D}}} D_{\mathrm{eff}}$.

Here $e$ is the elementary charge, $m_{\mathrm{e}}$ is the electron mass, and $f_{12}$ is the oscillator strength (CHIANTI gives $f_{12}=0.759476$ ).

Then we again set the limit on optically thin solutions $\tau_{0}(\mathrm{C}$ III $) \leq 0.3-$ this gives us the selection of pixels for which the $\mathrm{L} \alpha$ and $\mathrm{C}$ III lines are both optically thin, which justifies our implementation of the method deriving $T$ and $\xi$. This gives 30 points in total (see Fig. 1), for which the best-fit models were found as the best agreement between spectroscopic observations and computations. For each pixel the best model was selected in such a way that the C III synthetic intensity was closest to the observed intensity. The results are shown as histograms. In Fig. 5 we show the distribution of the kinetic temperature with a red dashed line: the studied prominence is hot, with a temperature of between 50000 and $200000 \mathrm{~K}$. The histogram in

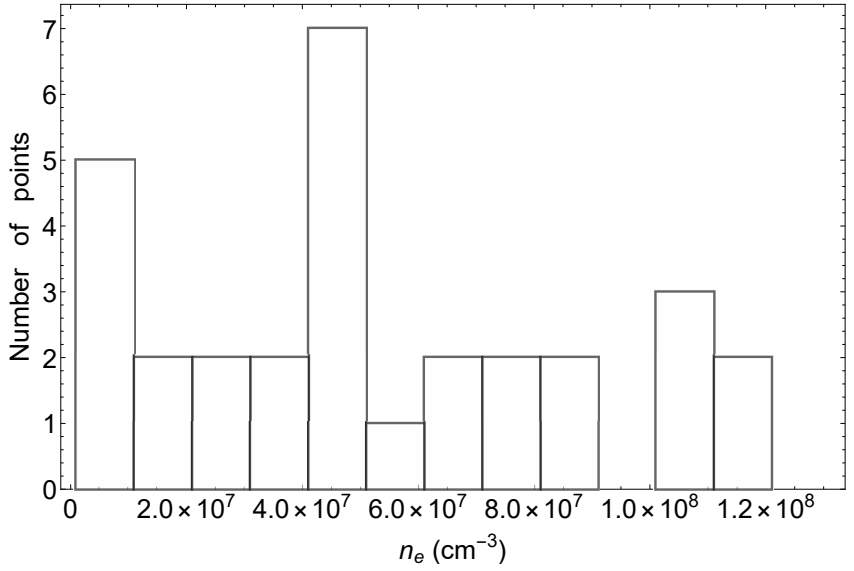

Fig. 15. Electron density distribution for all 30 selected points along the prominence in optically thin structures.

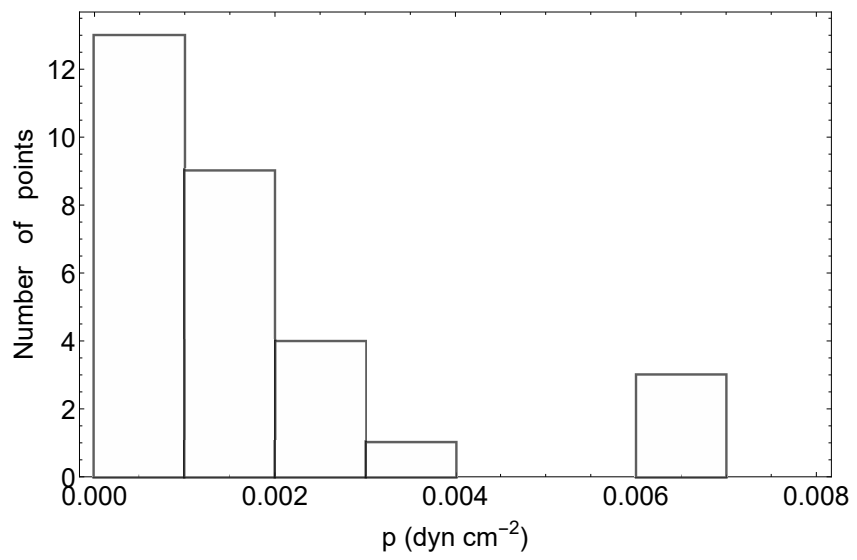

Fig. 16. Gas pressure distribution for all 30 selected points along the prominence in optically thin structures.

Fig. 6 shows the distribution of the microturbulent velocity with a red dashed line; the peak is at around $25 \mathrm{~km} \mathrm{~s}^{-1}$. Figure 13 shows the distribution of the effective thickness, which varies between $500 \mathrm{~km}$ and $50000 \mathrm{~km}$. Figure 14 reports the distribution of the radial flow velocity, which has a peak at $80 \mathrm{~km} \mathrm{~s}^{-1}$ and reaches up to $200 \mathrm{~km} \mathrm{~s}^{-1}$, while Fig. 15 shows the distribution of the electron density. The eruptive prominence is a lowdensity structure with an electron density of between $10^{7}$ and $10^{8} \mathrm{~cm}^{-3}$. The last histogram in Fig. 16 shows the distribution of the gas pressure. The gas pressure is low, with values of up to $0.007 \mathrm{dyn} \mathrm{cm}^{-2}$. To compare with the results of other similar studies, we mention Akmal et al. (2001) and Lee et al. (2009), who found densities of about $10^{6} \mathrm{~cm}^{-3}$ using the $\mathrm{OV}$ line ratio. Raymond \& Ciaravella (2004) found a range of densities of $5 \times 10^{5}-10^{7} \mathrm{~cm}^{-3}$ using the radiative and collisional excitation of the O VI doublet. Murphy et al. (2011) used both diagnostics to obtain densities of around $10^{7} \mathrm{~cm}^{-3}$. All these densities are somewhat lower than our typical values. However, Landi et al. (2010) found a temperature upper limit of $40000 \mathrm{~K}$ from the $\mathrm{L} \alpha$ line width, which is lower than our typical value of $10^{5} \mathrm{~K}$.

Figure 17 shows the relation between microturbulent velocity and kinetic temperature for the 30 optically thin points, as derived in Sect. 4. The points are strongly scattered around a mean value of about $25 \mathrm{~km} \mathrm{~s}^{-1}$. It is not evident that the microturbulent velocity increases with kinetic temperature, as was assumed in Paper I (see Appendix A) and observed by Parenti \& Vial (2007). We therefore added for comparison two examples with 


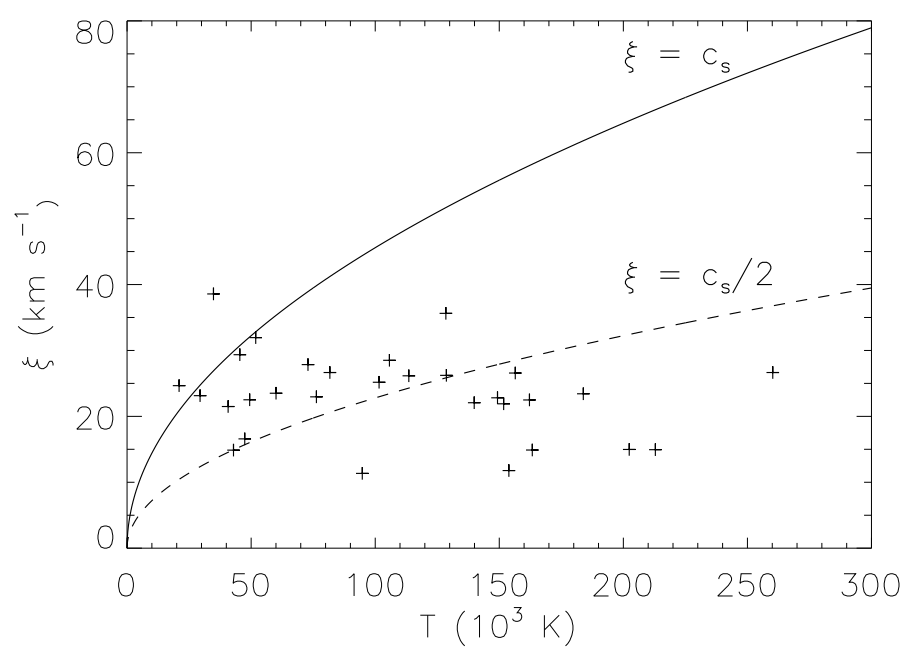

Fig. 17. Microturbulence as a function of kinetic temperature for 30 optically thin points. For comparison we added the solid curve, which presents the case where the microturbulence is equal to the sound speed, and the dashed curve for the case where the microturbulence is one-half of the sound speed, as assumed in Paper I.

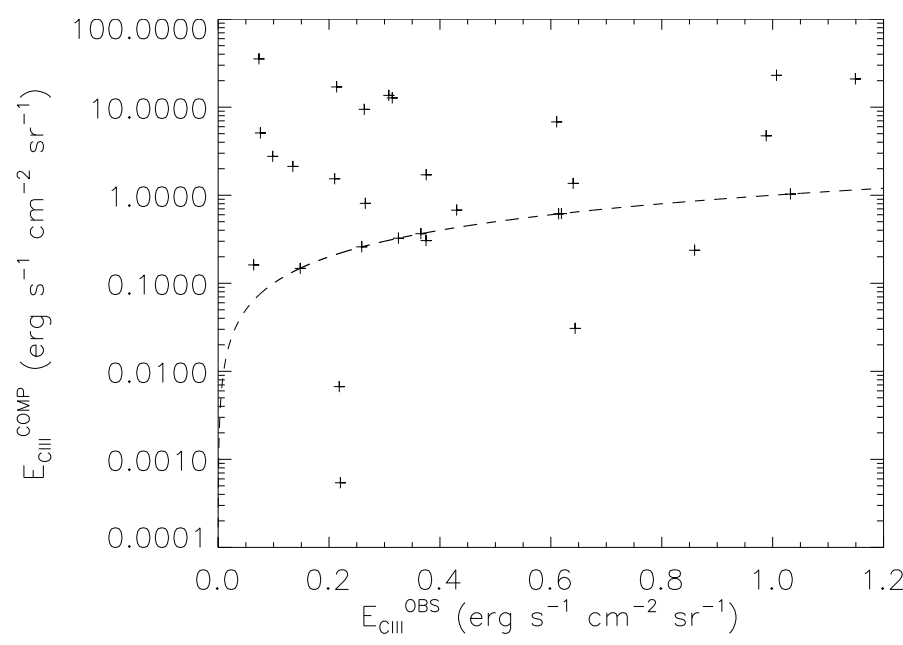

Fig. 18. Comparison between the integrated intensities of the C III line from spectroscopic observations and from best-fit models. The dashed line shows where the two integrated intensities are equal. Note that for the $y$-axis the logarithmic scale is used.

a microturbulence equal to one-half of the sound speed $c_{\mathrm{s}}$, as in Paper I (dashed curve), and the case where the microturbulence is equal to the sound speed (solid curve). Almost all points lie below the solid curve $\xi=c_{\mathrm{s}}$, and about one-third of the points lie below the dashed curve $\xi=c_{\mathrm{s}} / 2$. The sound speed was computed in the same way as in Appendix A of Paper I. The result clearly shows that the microturbulence is subsonic. However, that the nonthermal velocity is independent of temperature might also indicate that some other nonthermal broadening takes place. This could be an expansion of the eruptive prominence as we mentioned in Paper I; our current estimate of the expansion velocity is on the order of $10 \mathrm{~km} \mathrm{~s}^{-1}$.

Our hope was that the observed C III line intensity would lie in the range of $\mathrm{C}$ III line intensities synthesized from our best-fit models for each pixel, taking into account the data uncertainty (set to $40 \%$ in our case). However, the resulting plot in Fig. 18 is not very encouraging - only about one-third of the pixels satisfies our expectations, while the others show a relatively large scatter, most of them with computed intensities that are much

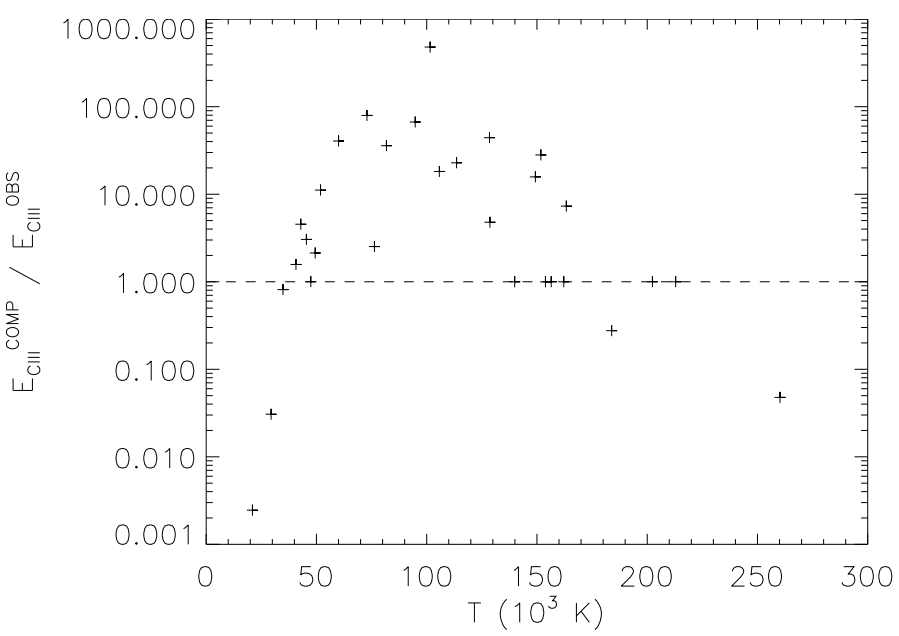

Fig. 19. Ratio of the best-fit integrated intensities of the C III best-fit models and from the spectroscopic observations as a function of kinetic temperature. Note that for $y$-axis the logarithmic scale is used.

higher than observed. However, to our surprise, this scatter does not seem to represent a random scatter due to the quality of UVCS data or to the way we fit these data with the non-LTE models. In Fig. 19 we plot the line ratio (computed over the observed intensity) versus the kinetic temperature and clearly see an almost unique trend, indicating that a certain physical reason is inherent in the differences between the observed and synthetic intensities. To our current understanding, the reason might be that we used the Dere (2007) ionization equilibrium for carbon, which neglects the photoionization processes. When we include the photoionization of the C III to C IV state, the C III population will be decreased and so will the synthetic intensity at relevant temperatures be (e.g., at around $80000 \mathrm{~K}$ ). For much lower temperatures, where the synthetic intensities steeply decrease below the value of the observed intensities, the lack of $\mathrm{C}$ III ions could be explained by the missing photoionization of $\mathrm{C}$ II that is formed at lower temperatures. The crossing point at around $50000 \mathrm{~K}$ represents the situation when the $\mathrm{C}$ II photoionization to $\mathrm{C}$ III could be compensated for by leakage of C III to C IV, meaning that the ionization equilibrium is close to that of Dere (2007). For high enough temperatures, the equilibrium should be close to the values reported by Dere (2007). A qualitatively similar picture follows from the full non-LTE radiative-transfer modeling of Avrett (2007), who used the multilevel/multi-ion carbon model to compute a realistic ionization equilibrium. We computed the photoionization rates for $\mathrm{C}$ II and C III ions, taking into account the prominence illumination in the far-UV. The resulting estimates show the importance of photoionizations for temperatures below $10^{5} \mathrm{~K}$, at electron densities typical for this erupting prominence. To prove the above scenario, we plan in a future paper to compute a detailed non-LTE ionization equilibrium and also to investigate the possible role of non-equilibrium ionization (see, e.g., Murphy et al. 2011). The latter can be quite important in our case because at low electron densities the ionization and recombination timescales are long.

\section{Discussion and conclusions}

This study is a continuation of Paper I, where we derived the physical parameters of the hot erupting prominence plasma embedded in the core of a CME by using SOHO/UVCS hydrogen $\mathrm{L} \alpha$ and SOHO/LASCO VL observations. All diagnostics with the $\mathrm{L} \alpha$ line has been based on the assumption that the 
microturbulent velocity is a constant fraction (one-half) of the sound speed (see Appendix A in Paper I). In order to check this assumption, we decided to study the C III line at $977 \AA$, which was simultaneously observed with UVCS. We note that for the $\mathrm{C}$ III line we only used raw data with radiometric calibration because the line is focused close to the detector edge where the standard calibration procedure, which includes other corrections, causes spurious line broadening. In this paper we combined the $\mathrm{L} \alpha$ and $\mathrm{C}$ III spectra to derive the kinetic temperature and the microturbulence from the line widths, which is feasible for two optically thin lines formed in the same plasma volume, as described by Tandberg-Hanssen (1995). We computed Doppler velocity shifts in the $\mathrm{L} \alpha, \mathrm{L} \beta$, and C III lines for the same sample of points chosen along the prominence (Paper I) in order to demonstrate that these lines are formed in the same moving plasma volume. We considered only solutions with positive microturbulent velocities and kinetic temperatures higher than $20000 \mathrm{~K}$, which conditions were satisfied in a total of 39 points.

We then used the same non-LTE code as described in Paper I to derive the physical parameters of the plasma in this subsample of 39 points. The input parameters were the kinetic temperature, the microturbulent velocity, and the height above the solar surface. Other input parameters such as the effective thickness, the radial flow velocity, and the electron density (proportional to gas pressure) were computed for the first time with a nonLTE code by achieving the best reproduction of the measured $\mathrm{L} \alpha$ and $\mathrm{L} \beta$ line intensities with a $40 \%$ accuracy of the absolute radiometric calibration. As the result, we also derived the optical thicknesses of the $\mathrm{L} \alpha$ and $\mathrm{L} \beta$ lines. We considered only solutions with $\tau_{0}(\mathrm{~L} \alpha) \leq 0.3$ (38 points). For each point we obtained a set of models within the prescribed $40 \%$ accuracy, and for each of the models, we computed the optical thickness and integrated intensity of the C III line using a two-level atom with the radiative excitation component. Neglecting the latter, this approximation is consistent within $10 \%$ with the CHIANTI multilevel C III model. We then searched for models that were optically thin in the $\mathrm{L} \alpha$ and $\mathrm{C}$ III lines, that is, $\tau_{0}(\mathrm{~L} \alpha) \leq 0.3$ and $\tau_{0}(\mathrm{C}$ III $) \leq 0.3$. In this way, we narrowed down the sample further to 30 points. The best-fit model for each point was then found as the best agreement between C III line observations and computations. The results are presented as histograms of the kinetic temperature, microturbulent velocity, effective thickness, radial flow velocity, electron density, and gas pressure. These results show that the prominence is composed of relatively hot plasma with low electron density and gas pressure, moving at lower radial flow velocities (peak at $80 \mathrm{~km} \mathrm{~s}^{-1}$ ) and with an effective thickness ranging from 500 to $50000 \mathrm{~km}$, and the results confirm to some extent our previous results presented in Paper I.

The microturbulence does not exhibit any increase with the kinetic temperature. From this it follows that the microturbulence is not a constant fraction of the sound speed, as we assumed in Paper I, and that the nonthermal broadening might also be due to expansion of the prominence flux tube. In the scatter plot of the computed versus observed integrated intensities of the C III line, we found that more than one-half of the points have higher computed intensities than observed. However, the plot showing the ratio of computed to observed C III intensities as a function of the kinetic temperature has an interesting behavior, which in our opinion has a realistic physical explanation. The discrepancy between observations and computations in the C III line is very likely due to photoionization processes that are neglected in the CHIANTI modeling. For temperatures lower than $50000 \mathrm{~K}$, the number of C III ions can be increased through photoionization of $\mathrm{C}$ II to $\mathrm{C}$ III in order to enhance the computed integrated intensity and make it consistent with the observations. Neglecting photoionization also leads to a higher optical thickness of the C III line, which might cause opacity broadening and consequently a spurious higher microturbulent velocity. For these optically thick points, the microturbulent velocity is therefore not correctly computed, and the kinetic temperature may likewise differ. At temperatures of between 50000 and $120000 \mathrm{~K}$, the number of $\mathrm{C} \mathrm{III} \mathrm{ions} \mathrm{can} \mathrm{be} \mathrm{reduced} \mathrm{with}$ respect to the CHIANTI results, causing a lower optical thickness and a lower line intensity. At still higher temperatures, the CHIANTI model probably is a good approximation.

In order to check our interpretation, we postpone a detailed non-LTE modeling of C III line formation to the next paper. This modeling will require solving a system of statistical equilibrium equations for at least the $\mathrm{C}$ II-C III-C IV ionic system, taking into account all important radiative and collisional processes. In particular, we will test the importance of photoionizations on the C III ionic population and compare the results with CHIANTI. However, the question of non-equilibrium ionization still remains. This has been recently studied by Murphy et al. (2011), and it might also affect our results.

Acknowledgements. S.J., P.H., and E.D. acknowledge support from the Czech Funding Agency through grant No. 16-18495S and from the AIAS through RVO67985815. S.J. acknowledges financial support from the Slovenian Research Agency (research core funding No. P1-0188). The work of R.S. was supported by the Agenzia Spaziale Italiana through contract ASI/INAF No. I/013/12/0-1. CHIANTI is a collaborative project involving George Mason University (USA), the University of Michigan (USA), and the University of Cambridge (UK). We are grateful to the anonymous referee for useful comments.

\section{References}

Akmal, A., Raymond, J. C., Vourlidas, A., et al. 2001, ApJ, 553, 922

Avrett, E. H. 2007, in The Physics of Chromospheric Plasmas, eds. P. Heinzel, I. Dorotovič, \& R. J. Rutten, ASP Conf. Ser., 368

Berrington, K. A., Burke, P. G., Dufton, P. L., \& Kingston, A. E. 1985, At. Data Nucl. Data Tables, 33, 195

Berrington, K. A., Burke, V. M., Burke, P. G., \& Scialla, S. 1989, J. Phys. B, 22, 665

Bryans, P., Badnell, N. R., Gorczyca, T. W., et al. 2006, ApJS, 167, 343

Curdt, W., Brekke, P., Feldman, U., et al. 2001, A\&A, 375, 591

Del Zanna, G., Dere, K. P., Young, P. R., Landi, E., \& Mason, H. E. 2015, A\&A, 582, A56

Dere, K. P. 2007, A\&A, 466, 771

Dere, K. P., Landi, E., Mason, H. E., Monsignori Fossi, B. C., \& Young, P. R. 1997, A\&AS, 125

Gontikakis, C., \& Vial, J.-C. 2016, A\&A, 590, A86

Habbal, S. R., Druckmüller, M., Morgan, H., et al. 2010, ApJ, 719, 1362

Heinzel, P., \& Anzer, U. 2001, A\&A, 375, 1082

Heinzel, P., \& Rompolt, B. 1987, Sol. Phys., 110, 171

Heinzel, P., Susino, R., Jejčič, S., Bemporad, A., \& Anzer, U. 2016, A\&A, 589, A128

Hubeny, I., \& Mihalas, D. 2015, Theory of Stellar Atmospheres (Princeton University Press)

Ko, Y.-K., Raymond, J. C., Gibson, S. E., et al. 2005, ApJ, 623, 519

Labrosse, N., Heinzel, P., Vial, J.-C., et al. 2010, Space Sci. Rev., 151, 243

Landi, E., Raymond, J. C., Miralles, M. P., \& Hara, H. 2010, ApJ, 711, 75

Lee, J.-Y., Raymond, J. C., Ko, Y.-K., \& Kim, K.-S. 2009, ApJ, 692, 1271

Martin, W., Sugar, J., Musgrove, A., \& Dalton, G. 1995, Version 1.0, NIST Standard Reference Database 61

Murphy, N. A., Raymond, J. C., \& Korreck, K. E. 2011, ApJ, 735, 17

Pagano, P., Mackay, D. H., \& Poedts, S. 2014, A\&A, 568, A120

Parenti, S. 2015, in Solar Prominences, eds. J.-C. Vial, \& O. Engvold, Astrophys. Space Sci. Lib., 415, 61

Parenti, S., \& Vial, J.-C. 2007, A\&A, 469, 1109

Raymond, J. C., \& Ciaravella, A. 2004, ApJ, 606, 159

Tachiev, G., \& Froese Fischer, C. 1999, J. Phys. B: At. Mol. Phys., 32, 5805

Tandberg-Hanssen, E. 1995, Science, 269, 111

Vial, J.-C., \& Engvold, O. 2015, Solar Prominences, Astrophys. Space Sci. Lib., 415

Webb, D. F. 2015, in Solar Prominences, eds. J.-C. Vial, \& O. Engvold, Astrophys. Space Sci. Lib., 415, 411 